\title{
ON PRIMITIVE ELEMENTS IN DIFFERENTIALLY ALGEBRAIC EXTENSION FIELDS
}

\author{
BY \\ A. BABAKHANIAN
}

It is well known that if $F$ is a field of characteristic zero and $K=F\left(\alpha_{1}, \ldots, \alpha_{n}\right)$ is a finite algebraic extension of $F$, then $K$ contains a primitive element, i.e. an element $\alpha$ such that $F\left(\alpha_{1}, \ldots, \alpha_{n}\right)=F(\alpha)$. Moreover, by means of Galois theory, it is possible to characterize those elements of the extension field which are primitive.

In the case of finite differentially algebraic extensions the theorem without further restrictions is false. Let $Q$ be the field of rational numbers and $\delta$ the usual derivation, i.e., $\delta q=0$ for every $q \in Q$. Let $c_{1}, \ldots, c_{n}$ be algebraically independent complex numbers over $Q$. If $\left(Q\left\langle c_{1}, \ldots, c_{n}\right\rangle, \delta\right)$ is the differentially algebraic extension of $Q$ where $\delta c=0$ for every $c \in Q\left\langle c_{1}, \ldots, c_{n}\right\rangle$, then the underlying set of $Q\left\langle c_{1}, \ldots, c_{n}\right\rangle$ is identical with that of $Q\left(c_{1}, \ldots, c_{n}\right)$, whence it is clear that there is no element $c \in Q\left\langle c_{1}, \ldots, c_{n}\right\rangle$ such that $Q\left\langle c_{1}, \ldots, c_{n}\right\rangle=Q\langle c\rangle$. Kolchin [2] (also [5, p. 52]) has shown the existence of primitive elements in the case where the differential field $F\left\langle\alpha_{1}, \ldots, \alpha_{n}\right\rangle$ has one derivation operator and the field $F$ has an element $f$ such that $\delta f \neq 0\left({ }^{1}\right)$. The differential fields $\left(F\left\langle x_{0}, \ldots, x_{p}\right\rangle, D\right)$ considered in this paper are differentially algebraic over $F$, but $F$ does not contain nonconstant elements. We prove the existence of primitive elements in the case where the derivation operator satisfies the conditions

$$
\begin{gathered}
D f=0 \quad \text { for every } f \in F, \quad D x_{0}=1, \\
x_{0} \cdot x_{1} \cdots \cdots x_{k-1} D x_{k}=1 \quad \text { for } 0<k \leqq p .
\end{gathered}
$$

An example of such a differential field is $\left(C\left\langle e^{z}, z, \log z, \log \log z\right\rangle, \delta\right)$ where $C$ is the field of complex numbers, $\delta=e^{-z} D$ and $D$ is the usual derivation of functions of a complex variable, i.e., $\delta e^{z}=e^{-z} D e^{z}=1, \quad \delta \log z=e^{-z} D \log z=\left(e^{z} \cdot z\right)^{-1}$, $\delta \log \log z=e^{-z} D \log \log z=\left(e^{z} \cdot z \cdot \log z\right)^{-1}$.

In the sequel, for differentially algebraic extension fields which satisfy conditions (1) not only do we establish the existence of primitive elements, but we give explicit formulas for such elements. In $\$ \$ 7$ and 8 we apply these formulas to the asymptotic theory of ordinary differential equations. More precisely, in [6] W. Strodt introduced the concept of the "principal monomials" and "principal solutions" for a certain class of differential equations whose coefficients belong to a logarithmic domain. In [8] Strodt characterized the principal monomials by the concept of

Received by the editors February 21, 1967 and, in revised form, June 19, 1967.

(1) In the partial case (more than one derivation operator) $F$ must contain a set of elements whose Jacobian does not vanish (Kolchin $[2, \$ 4]$ ). 
stability. S. Bank [1] investigated all the logarithmic monomials at which an $n$th order differential polynomial of a certain class is unstable; such logarithmic monomials were called "critical monomials" of the differential polynomial. The algorithm which produced the principal monomials in [6] and the critical monomials in [1] consists essentially of the repeated applications of the transformation $x=e^{u}, y=v e^{m u}$. The effectiveness of this transformation depends upon two crucial lemmas ([6, Lemma 61] and [1, Lemma 13]), to the effect that whenever a transformation $x=e^{u}, y=v e^{m u}$ is applied to a homogeneous, isobaric differential polynomial of positive weight $W$, with constant coefficients, the transformed differential polynomial always effectively involves at least one term whose weight is less than $W$, unless the differential polynomial is of the form $c Y^{d-w} \cdot Y^{\prime w}$ and $m=0$. In this note we generalize these lemmas and prove them with the aid of a result on the transcendence degree of differential field extensions (Theorem 7.1 below) $\left({ }^{2}\right)$.

I am grateful to W. Strodt for his help in the preparation of this manuscript.

1. Preliminaries. This section contains some elementary results on differential field extensions. All differential fields considered here are of characteristic zero. The notations are the same as in [3], [4], and [5]. The differential field defined over a field $G$ by a derivation $\delta$ will be denoted by $(G, \delta)$.

LEMMA 1.1. Let $(H, \delta)$ be a subdifferential field of $(G, \delta)$. Suppose the subfield $C$ of elements of $G$ annihilated by $\delta$ is contained in $H$. If $\alpha \in G$ is such that $\delta \alpha \in H$, then either $\alpha$ is transcendental over $H$ or $\alpha \in H$.

Proof. Follows along the same lines as the proof of Lemma 3.9, Kaplansky [4].

LEMMA 1.2. Let $(H, \delta)$ be a differential field and $(H\langle\alpha\rangle, \delta)$ a differential extension field of $(H, \delta)$ such that $\alpha$ is transcendental over $H$ and $\delta \alpha=1\left({ }^{3}\right)$. Then there is no element in $H\langle\alpha\rangle$ whose derivative is $\alpha^{-1}$.

Proof. Since $\delta \alpha=1 \in H$, the underlying sets of $H\langle\alpha\rangle$ and $H(\alpha)$ are identical. Thus every $g \in H\langle\alpha\rangle, g \neq 0$, can be written in the form $\alpha^{n}(P / Q)$ where $n$ is an integer, and $P$ and $Q$ are polynomials in $\alpha$ with coefficients in $H$ such that $P(0) \neq 0, Q(0) \neq 0$. The integer $n$ is uniquely determined by $g$; by direct calculation $\delta\left(\alpha^{n}(P / Q)\right) \neq \alpha^{-1}$.

We will need the following well-known lemma:

LEMMA 1.3. Let $(C\langle\alpha\rangle, \delta)$ be a differential field where $C$ is the subfield of elements of $C\langle\alpha\rangle$ annihilated by $\delta$. If $C\langle\alpha\rangle$ has transcendence degree $p+1$ over $C$, then $\alpha, \delta \alpha, \ldots, \delta^{p} \alpha$ are algebraically independent over $C$. Moreover

$$
C\langle\alpha\rangle=C\left(\alpha, \delta \alpha, \ldots, \delta^{p+1} \alpha\right)
$$

$\left({ }^{2}\right)$ A proof of the case $m \neq 0$ in the setting of graduated logarithmic fields, independently of ours, is given by Strodt [8].

(3) Such a differential field extension can always be constructed. See Corollary 1 of Theorem 39 , page 124, Vol. 1 of [9]. 
2. The logarithmic differential fields. In this section $K$ is a differential field with derivation $D$. $K$ contains a distinguished sequence $x_{0}, x_{1}, \ldots$ of elements called a logarithmic sequence such that $D x_{0}=1$ and $x_{0} \cdot x_{1} \cdots \cdots x_{p-1} D x_{p}=1$ for $p=$ $1,2, \ldots\left({ }^{4}\right) . C=\{c: c \in K, D c=0\}$. For any $p \geqq 0$ the subdifferential field

$$
\left(C\left(x_{0}, x_{1}, \ldots, x_{p}\right), D\right)
$$

will be called a logarithmic differential field.

LeMMA 2.1. Let $F_{p}=C\left(x_{0}, x_{1}, \ldots, x_{p}\right)$ for each nonnegative integer $p$, and $F_{-1}=C$. Then for $p=0,1,2, \ldots, x_{p} \notin F_{p-1}$.

Proof. By induction on $p$. Since $D x_{0}=1 \neq 0$, for $p=0$ we have $x_{p}=x_{0} \notin C=F_{p-1}$. Suppose for the nonnegative integer $q, x_{q} \notin F_{q-1}$. Since $\left(F_{q-1}, D\right)$ is a differential field and $D x_{q}=\left(x_{0} \cdot x_{1} \cdots \cdots x_{q-1}\right)^{-1} \in F_{q-1}$, by Lemma 1.1, $x_{q}$ is transcendental over $F_{q-1}$. Let $\delta_{q}=x_{0} \cdot x_{1} \cdots \cdots x_{q-1} D$, then $\left(F_{q-1}, \delta_{q}\right)$ is also a differential field. Since $x_{q}$ is transcendental over $F_{q-1}$ and $\delta_{q} x_{q}=1$, by Lemma 1.2, there is no element $y$ in $\left(F_{q-1}\left\langle x_{q}\right\rangle, \delta_{q}\right)$ such that $\delta_{q} y=x_{q}^{-1}$. Since $\delta_{q} x_{q+1}=x_{q}^{-1}, x_{q+1} \notin F_{q-1}\left\langle x_{q}\right\rangle$ $=F_{q}$. This completes the induction.

LEMMA 2.2. $x_{0}, x_{1}, \ldots$ are algebraically independent over $C$.

Proof. Follows from Lemmas 1.1 and 2.1 .

3. Partial order in $\left(C\left(x_{0}, x_{1}, \ldots, x_{p}\right), D\right)$. Let $K, C$, and the sequence $x_{0}, x_{1}, \ldots$ be as in $\$ 2$. We will introduce a partial order in the subfield $F_{p}=C\left(x_{0}, x_{1}, \ldots, x_{p}\right)$ of $K$ as follows. Let

$$
V_{p}=\left\{x_{0}^{i_{0}} \cdot x_{1}^{i_{1}} \cdots \cdots x_{p}^{i_{p}}:\left(i_{0}, i_{1}, \ldots, i_{p}\right) \in Z^{p}\right\}
$$

where $Z$ is the ring of integers. $V_{p}$ is a subgroup of the multiplicative group of $F_{p}$. Let $\mathscr{N}_{p}=\left\{c v: c \in C-\{0\}, v \in V_{p}\right\}$. Let $M=a x_{0}^{m_{0}} \cdot x_{1}^{m_{1}} \cdots \cdots x_{p}^{m_{p}}$ and $N=b x_{0}^{n_{0}} \cdot x_{1}^{n_{1}}$. $\cdots x_{p}^{n}$ be elements of $\mathscr{N}_{p}$. We will write $M<N$ if $m_{0}<n_{0}$, or for some natural number $q, 0<q \leqq p, m_{k}=n_{k}$ for $k=0,1, \ldots, q-1$ and $m_{q}<n_{q}$. If $\left(m_{0}, m_{1}, \ldots, m_{p}\right)$ $=\left(n_{0}, n_{1}, \ldots, n_{p}\right)$ we write $M \approx N$. If $f \in C\left[x_{0}, x_{1}, \ldots, x_{p}\right], f \neq 0$, it can be written in the form $f=\sum_{i=1}^{n} c_{i} M_{i}$ where $c_{i} \in C-\{0\}$ and $M_{i} \in V_{p}$ such that $M_{i} \neq M_{j}$ if $i \neq j$. For some positive integer $s, 1 \leqq s \leqq n, c_{i} M_{i}<c_{s} M_{s}$ for $i \neq s$. We will call $c_{s} M_{s}$ the dominating monomial of $f$. If $g \in F_{p}-\{0\}$ then $g$ can be written $g=\sum c_{i} M_{i} / \sum b_{j} N_{j}$ where $c_{i}, b_{j} \in C-\{0\}$ and $M_{i}, N_{j} \in V_{p}$. If $c_{s} M_{s}$ is the dominating monomial of $\sum c_{i} M_{i}$ and $b_{t} N_{t}$ is the dominating monomial of $\sum b_{j} N_{j}$ we say $g^{*}=\left(c_{s} M_{s}\right)\left(b_{t} N_{t}\right)^{-1}$ is the dominating monomial of $g$. If $g, h \in F_{p}-\{0\}$ we write $g<h$ if $g^{*}<h^{*}$, and $g \sim h$ if $g^{*}=h^{*}$. We extend this definition of order to $F_{p}$ by setting $0<g$ for every $g \in F_{p}-\{0\}\left({ }^{5}\right)$. It is clear now that if $f, g, h, k \in F_{p}$,

(4) The notion of logarithmic sequences was introduced by Strodt (cf. [6] and [8]).

(5) $F_{p}$ with the partial order < is a field with asymptotic order (cf. Strodt [7]). 
(i) if $f<g$, then $f \neq g$;

(ii) $0<1$;

(iii) if $f<g$ and $h \neq 0$, then $f h<g h$;

(iv) if $f<g$ and $h<k$, then $f h<g k$;

(v) if $f<g$ and $h<g$, then $f+h<g$;

(vi) if $f<g$ and $h<g$, then $f<g+h$;

(vii) if $f \sim g$ then $f-g<g$ and conversely;

(viii) if $f \sim g$ and $g \sim h$, then $f \sim h$;

(ix) if $f \sim g$ and $h \sim k$, then $f h \sim g k$;

(x) if $f \sim g$ then $f \neq 0$ and $g \neq 0$.

Definition 3.1. Let

$$
f=\frac{\sum b_{i} x_{0}^{i_{0}} \cdot x_{1}^{i_{1}} \cdots \cdots x_{p}^{i_{p}}}{\sum c_{f} x_{0}^{j_{0}} \cdot x_{1}^{j_{1}} \cdots \cdots x_{p}^{j_{p}}} \in F_{p}
$$

Define $E f \in F_{p+1}$ to be

$$
E f=\frac{\sum b_{1} x_{1}^{i_{0}} \cdot x_{2}^{i_{1}} \cdots \cdots x_{p+1}^{i_{p}}}{\sum c_{f} x_{1}^{j_{0}} \cdot x_{2}^{j_{1}} \cdots \cdots x_{p+1}^{j_{p}}}
$$

LEMMA 3.1. Let $f, g \in F_{p}$, then

(i) $f<g$ implies $E f<E g$.

(ii) $f \sim g$ implies $E f \sim E g$.

Proof. Follows from the definitions.

4. The functions $S_{i, j}(m)$.

Definition 4.1. Let $m$ be a variable; define

$$
S_{k, k}(m)=m(m-1)(m-2) \cdots(m-k+1) \text { if } k>0,
$$

and

$$
S_{k, k-j}(m)=(1 / j !) S_{k, k}^{(j)}(m) \text { if } j>0,
$$

where $S_{k, k}^{(j)}(m)$ is the jth derivative of $S_{k, k}(m)$. We will make the convention that $S_{0,0}(m)=1$.

It is clear that $S_{i, j}(m)$ is the elementary symmetric function of degree $j$ in $m, m-1$, $\ldots, m-i+1$. Thus

$$
\sum_{j=0}^{k} x^{j} S_{k, k-j}(m)=(x+m)(x+m-1) \cdots(x+m-k+1)
$$

(cf. $[6, \S 58])$. We remark that $S_{k, 0}(m)=1$ and $S_{k, \gamma}(m)=0$ if $j<0$.

LEMMA 4.1. If $k \geqq 1, m=(-1)^{k+1} \cdot k \cdot S_{k, k}(m)-(-1)^{k} \cdot m \cdot \sum_{j=1}^{k-1}(-1)^{j} S_{k, k-f}(m)$. 
Proof. By (1) above $\sum_{j=0}^{k}(-1)^{j} S_{k, k-j}(m)=(m-1)(m-2) \cdots(m-k)$. Therefore

$$
\begin{aligned}
m \sum_{j=1}^{k-1}(-1)^{j} S_{k, k-j}(m) & =m\left[(m-1)(m-2) \cdots(m-k)-S_{k, k}(m)-(-1)^{k} S_{k, 0}(m)\right] \\
& =m\left[(m-1) \cdots(m-k)-m(m-1) \cdots(m-k+1)+(-1)^{k+1}\right] \\
& =m\left[(m-1) \cdots(m-k+1)(m-k-m)+(-1)^{k+1}\right] \\
& =-k S_{k, k}(m)+(-1)^{k+1} m .
\end{aligned}
$$

5. The elements $\left(x_{0} \cdot x_{1} \cdots \cdots x_{k}\right)^{m}$ of $\left(C\left(x_{0}, x_{1}, \ldots, x_{p}\right), D\right)$. Here and in the next section $m$ is a nonzero integer.

DefinItion 5.1. Let $E_{0}=E$ where $E$ is as in Definition 3.1, and for the positive integer $p, E_{p}=\left[(m-p+1)+x_{0} D\right] E_{p-1}$.

LEMMA 5.1. Let $V=V\left(x_{0}, x_{1}, \ldots, x_{k}\right) \in C\left(x_{0}, x_{1}, \ldots, x_{p}\right), k<p$. Then $x_{0} D E V$ $=E D V$.

Proof.

$$
\begin{aligned}
x_{0} D E V\left(x_{0}, x_{1}, \ldots, x_{k}\right) & =x_{0} D V\left(x_{1}, x_{2}, \ldots, x_{k+1}\right) \\
& =x_{0}\left[\frac{1}{x_{0}} \frac{\partial E V}{\partial x_{1}}+\frac{1}{x_{0} x_{1}} \frac{\partial E V}{\partial x_{2}}+\cdots+\frac{1}{x_{0} x_{1} \cdots x_{k}} \frac{\partial E V}{\partial x_{k+1}}\right] \\
& =\frac{\partial E V}{\partial x_{1}}+\frac{1}{x_{1}} \frac{\partial E V}{\partial x_{2}}+\cdots+\frac{1}{x_{1} \cdot x_{2} \cdots \cdots x_{k}} \frac{\partial E V}{\partial x_{k+1}} \\
& =E D V .
\end{aligned}
$$

Let $T_{k}=\left(x_{0} \cdot x_{1} \cdots \cdots x_{k}\right)^{m}$ where $m$ is a nonzero integer, then:

\section{COROLlary.}

$$
\begin{aligned}
E_{p} T_{k} & =\left(m-p+1+x_{0} D\right)\left(m-p+2+x_{0} D\right) \cdots\left(m-1+x_{0} D\right)\left(m+x_{0} D\right) E T_{k} \\
& =\sum_{i=0}^{p} S_{p, i}(m) E D^{p-i} T_{k} .
\end{aligned}
$$

Proof. By straightforward calculation and Lemma 5.1.

LEMMA 5.2( $\left.{ }^{6}\right) . D^{p} T_{0}=S_{p, p}(m) x_{0}^{m-p}$ and for positive integers $p$ and $k, D^{p} T_{k}$ $=x_{0}^{m-p} E_{p} T_{k-1}$.

Proof. By induction on $p . D^{0} T_{k}=T_{k}=x_{0}^{m} E_{0} T_{k-1}$ by the definitions of $T_{k}$ and $E_{0}$. Suppose $D^{p-1} T_{k}=x_{0}^{m-p+1} \cdot E_{p-1} T_{k-1}$. Then

$$
D^{p} T_{k}=x_{0}^{m-p}\left[(m-p+1)+x_{0} D\right] E_{p-1} T_{k-1}=x_{0}^{m-p} E_{p} T_{k-1}
$$

by the definition of $E_{p} T_{k-1}$.

$\left({ }^{6}\right)$ This is a special case of $[8$, Lemma $66(\mathrm{c})]$. 
LEMMA 5.3. For any pair of integers $(p, q)$ such that $0 \leqq p<q, D^{q} T_{k}<D^{p} T_{k}$ if $D^{p} T_{k} \neq 0$.

Proof. If $D^{a} T_{k}=0$, then $D^{a} T_{k}<D^{p} T_{k}$. Suppose $D^{a} T_{k} \neq 0$. By Lemma 5.2,

$$
D^{p} T_{k}=x_{0}^{m-p} E_{p} T_{k-1} \text { and } D^{a} T_{k}=x_{0}^{m-q} E_{q} T_{k-1} .
$$

Since $E_{p} T_{k-1}$ and $E_{q} T_{k-1}$ are elements of $C\left(x_{1}, x_{2}, \ldots, x_{k}\right)$ and $m-q<m-p$, it follows from the definition of $<$ that $D^{a} T_{k}<D^{p} T_{k}$.

Notation. For $f \in C\left(x_{0}, x_{1}, \ldots, x_{k}\right)$, by $\partial_{j} f$ we will mean the formal partial derivative of $f$ with respect to $x_{j}$.

LEMMA 5.4. For any pair of integers $(p, q)$ such that $0 \leqq p<q, \partial_{n} D^{q} T_{k}<\partial_{n} D^{p} T_{k}$ if $\partial_{n} D^{p} T_{k} \neq 0$ and $0 \leqq n \leqq k$.

Proof. Similar to the proof of Lemma 5.3.

6. The differential field $\left(C\left\langle T_{p}\right\rangle, D\right)$. In this section we will show that the transcendence degree of $\left(C\left\langle T_{p}\right\rangle, D\right)$ is $p+1$ over $C$.

Notation. In the sequel the minor of $\partial_{p} D^{i} T_{p-1}$ in the Jacobian determinant

$$
\frac{\partial\left(T_{p-1}, D T_{p-1}, \ldots, D^{p} T_{p-1}\right)}{\partial\left(x_{0}, x_{1}, \ldots, x_{p}\right)}
$$

will be denoted by $A_{i}$.

LEMMA 6.1. Suppose the Jacobian determinant

$$
J_{p-1}=\frac{\partial\left(T_{p-1}, D T_{p-1}, \ldots, D^{p-1} T_{p-1}\right)}{\partial\left(x_{0}, x_{1}, \ldots, x_{p-1}\right)} \neq 0,
$$

then $A_{i}<J_{p-1}$ for $i=0,1,2, \ldots, p-1$.

Proof. Write $D^{i} T_{p-1}=x_{0}^{m-i} E_{i} T_{p-2}$, by Lemma 5.2, and write $J_{p-1}$ and $A_{i}$ in the determinant form. By direct calculation

$$
J_{p-1} \sim d x_{0}^{p[m-(p-1) / 2]-1} \cdot x_{1}^{k_{1}} \cdot x_{2}^{k_{2}} \cdots \cdots x_{p-1}^{k_{p}-1} \quad \text { with } d \in C-\{0\},
$$

and either $A_{i}=0$ or

$$
A_{i} \sim c x_{0}^{p[m-(p-1) / 2]-1-(p-i)} \cdot x_{1}^{j_{1}} x_{2}^{j_{2}} \cdots \cdots x_{p-1}^{j_{p}-1}
$$

where $k_{1}, k_{2}, \ldots, k_{p-1}, j_{1}, j_{2}, \ldots, j_{p-1}$ are integers. $A_{i}<J_{p-1}$ for $i=0,1, \ldots, p-1$ by comparing the right sides of (3) and (4).

COROLLARY. If $J_{p-1} \neq 0, \sum_{i=1}^{p} m E_{0} A_{i} \sim m E_{0} J_{p-1}$.

Proof. Follows from Lemma 6.1 and the identity $A_{p}=J_{p-1}$.

LEMMA 6.2. If $J_{p-1} \neq 0$, then

$$
A=\sum_{i=0}^{p}(-1)^{i} D^{i} T_{p-1} A_{i}<T_{p-1} \cdot J_{p-1}
$$


Pronf. By the properties (iii) and (iv) of $\S 3$, and Lemmas 5.3 and 6.1,

$$
(-1)^{i} D^{i} T_{p-1} A_{i}<T_{p-1} J_{p-1} \quad \text { for } i=0, \ldots, p-1
$$

and

$$
(-1)^{p} D^{p} T_{p-1} \cdot A_{p}=(-1)^{p} D^{p} T_{p-1} \cdot J_{p-1}<T_{p-1} \cdot J_{p-1}
$$

by Lemma 5.3 and the property (iv) of $\S 3$. Therefore by (v) of $\S 3, A<T_{p-1} \cdot J_{p-1}$.

Corollary. $E_{0} A<E_{0} T_{p-1} \cdot E_{0} J_{p-1}$.

Proof. By Lemma 3.1.

LEMMA 6.3. Let $\bar{B}_{p}=\sum_{i=1}^{p} m E_{0} A_{i}$ and $\bar{B}=\sum_{i=1}^{p}(-1)^{i} \cdot i \cdot S_{i, i}(m) B_{i}$ where $B_{i}$ is the minor of $\partial_{0} E_{i} T_{p-1}$ in the Jacobian determinant

$$
\frac{\partial\left(E_{0} T_{p-1}, E_{1} T_{p-1}, \ldots, E_{p} T_{p-1}\right)}{\partial\left(x_{0}, x_{1}, \ldots, x_{p}\right)}
$$

then $\bar{B}_{p}=\bar{B}$.

Proof. $\bar{B}_{p}$ and $\bar{B}$ may be viewed as the expansions of two $(p+1) \times(p+1)$ determinants by the minors of the first columns. These first columns are respectively $\left(0,-m,+m, \ldots,(-1)^{p} m\right)^{t}$ and $\left(0,-S_{1,1}(m),-2 S_{2,2}(m), \ldots,-p S_{p, p}(m)\right)^{t}$. We introduce the $(p+1) \times(p+1)$ determinants $\bar{C}_{0}, \bar{C}_{1}, \ldots, \bar{C}_{p}$ recursively as follows. Define $\bar{C}_{0}=\bar{B}$ and define $\bar{C}_{k+1}$ as the determinant obtained from $\bar{C}_{k}$ by adding $-S_{k+1, k+2-j}(m)$ times the $j$ th row of $\bar{C}_{k}, j=1,2, \ldots, k+1$, to the $(k+2)$ th row of $\bar{C}_{k}$. Obviously $\bar{B}=\bar{C}_{0}=\bar{C}_{1}=\cdots=\bar{C}_{p}$. Evidently $\bar{C}_{0}$ has the form of $\bar{B}_{p}$ in the first row, and the form of $B$ in the remaining rows. Suppose now that for some $k$ in $\{0,1, \ldots, p-1\}, \bar{C}_{k}$ has the form of $\bar{B}_{p}$ in the first $k+1$ rows and the form of $\bar{B}$ in the remaining rows. By the above construction $\bar{C}_{k+1}$ has the form of $\bar{B}_{p}$ in the first $k+2$ rows. In fact, the $(k+2)$ th row of $\bar{C}_{k+1}$ is

$$
\begin{aligned}
\left(\left\{-(k+1) S_{k+1, k+1}(m)-m \sum_{i=1}^{k}(-1)^{i} S_{k+1, k+1-i}(m)\right\},\right. & \partial_{1} E_{0} D^{k+1} T_{p-1}, \ldots, \\
& \left.\partial_{p} E_{0} D^{k+1} T_{p-1}\right) .
\end{aligned}
$$

Hence the first entry in this row is

$$
\begin{array}{r}
(-1)^{k+1}\left\{(-1)^{k+2} \cdot(k+1) S_{k+1, k+1}(m)-(-1)^{k+1} m \sum_{i=1}^{k}(-1)^{i} S_{k+1, k+1-i}(m)\right\} \\
=(-1)^{k+1} m \text { by Lemma 4.1. }
\end{array}
$$

The remaining entries of this row have the asserted form by the Corollary of Lemma 5.1 .

Corollary. Suppose $J_{p-1} \neq 0$, then $\bar{B} \sim m E_{0} J_{p-1}$.

Proof. Follows from the Corollary of Lemma 6.1 and Lemma 6.3. 
LEMMA 6.4. If $J_{p-1} \neq 0$, then

$$
J_{p}=\frac{\partial\left(T_{p}, D T_{p}, \ldots, D^{p} \dot{T}_{p}\right)}{\partial\left(x_{0}, x_{1}, \ldots, x_{p}\right)} \sim m x_{0}^{(p+1)[m-p / 2]-1} \cdot E_{0} T_{p-1} \cdot E_{0} J_{p-1} .
$$

Proof. Write the Jacobian $J_{p}$ in the determinant form, then

$$
J_{p}=x_{0}^{(p+1)[m-p / 2)]-1} \cdot\{m \tilde{B}+\widetilde{C}\}
$$

where $\widetilde{B}$ and $\widetilde{C}$ are $(p+1) \times(p+1)$ determinants whose expansions in the minors of the first columns are respectively $\sum_{i=0}^{p}(-1)^{i} E_{i} T_{p-1} B_{i}$ and $\sum_{i=0}^{p}(-1)^{i+1} \cdot i \cdot E_{i} T_{p-1} B_{i}$. We assert that

$$
\widetilde{B}=E_{0} \sum_{i=0}^{p}(-1)^{i} D^{i} T_{p-1} A_{i}
$$

This can be verified by applying to $\widetilde{B}$ the same row operations as were applied to $\bar{B}$ in Lemma 6.3. Each column of $\widetilde{B}$, except the first, is thereby transformed into the corresponding column of a determinant $E_{0} A$ whose expansion in the minors of the first column is the right side of (5). The verification that the first column of $\widetilde{B}$ is transformed into the first column of $E_{0} A$ is done inductively as in Lemma 6.3 and turns upon the identity

$$
E_{k+1} T_{p-1}-\sum_{j=1}^{k+1} S_{k+1, k+2-j}(m) E_{0} D^{j-1} T_{p-1}=E_{0} D^{k+1} T_{p-1}
$$

which follows from the Corollary of Lemma 5.1. As for $\widetilde{C}, \widetilde{C}$ can be written

$$
\tilde{C}=\left(E_{0} T_{p-1}\right) \bar{B}+\sum_{i=0}^{p}(-1)^{i+1} \cdot i \cdot \sum_{j=0}^{i} S_{i, j}(m)\left(E_{0} D^{i-j} T_{p-1}\right) B_{i} .
$$

It can be shown by the same reduction as employed in Lemma 6.3 that the second term in the right side of (6) is $<E_{0} T_{p-1} E_{0} J_{p-1}$. It now follows from the Corollary of Lemma 6.3 that $\widetilde{m} \sim m E_{0} T_{p-1} E_{0} J_{p-1}$. Thus, by the Corollary of Lemma 6.2 , $m \widetilde{B}+\widetilde{C} \sim m E_{0} T_{p-1} \cdot E_{0} J_{p-1}$. This establishes the lemma.

COROLLARY. If $J_{p-1} \neq 0$, then $J_{p} \neq 0$.

THEOREM 6.1. The differential field $\left(C\left\langle T_{p}\right\rangle, D\right)$ is of transcendence degree $p+1$ over $C$.

Proof. Since $\left(C\left\langle T_{p}\right\rangle, D\right)$ as a field is contained in the field $C\left(x_{0}, x_{1}, \ldots, x_{p}\right)$, the transcendence degree of $C\left\langle T_{p}\right\rangle$ over $C$ is at most $p+1$. It is enough, therefore, to show that $T_{p}, D T_{p}, \ldots, D^{p} T_{p}$ are algebraically independent over $C$. By induction on $p$ and the Corollary of Lemma 6.4 , it is enough to show that the Jacobian $J_{0} \neq 0$. This by direct calculation is $m x_{0}^{m-1} \neq 0$.

THEOREM 6.2. (i) $\left(C\left(x_{0}, x_{1}, \ldots, x_{p}\right), D\right)=\left(C\left\langle x_{0} \cdot x_{1} \cdots \cdots x_{p}\right\rangle, D\right)$,

(ii) $\left(C\left(x_{0}, x_{1}, \ldots, x_{p}\right), D\right)=\left(C\left\langle x_{p}\right\rangle, D\right)$. 
Proof of (i). By Theorem 6.1, $\left(C\left\langle x_{0} \cdot x_{1} \cdots \cdots x_{p}\right\rangle, D\right)$ is of transcendence degree $p+1$ over $C$. Thus $\left(C\left(x_{0}, x_{1}, \ldots, x_{p}\right), D\right)$ is an algebraic extension of

$$
\left(C\left\langle x_{0} \cdot x_{1} \cdots \cdot x_{p}\right\rangle, D\right) \text {. }
$$

By Lemma 1.1, it is enough to show $D x_{0}, D x_{1}, \ldots, D x_{p}$ are in

$$
\left(C\left\langle x_{0}, x_{1}, \ldots, x_{p}\right\rangle, D\right) \text {. }
$$

$D x_{0}=1$; therefore $x_{0} \in\left(C\left\langle x_{0} \cdot x_{1} \cdots \cdots x_{p}\right\rangle, D\right)$. Suppose

$$
x_{0}, x_{1}, \ldots, x_{k-1} \in\left(C\left\langle x_{0} \cdot x_{1} \cdots \cdots x_{p}\right\rangle, D\right) .
$$

Since $D x_{k}=\left(x_{0} \cdot x_{1} \cdots \cdots x_{k-1}\right)^{-1}$, then $x_{k} \in\left(C\left\langle x_{0} \cdot x_{1} \cdots x_{p}\right\rangle, D\right)$.

Proof of (ii). Since $D x_{p}=\left(x_{0} \cdot x_{1} \cdots \cdots x_{p-1}\right)^{-1}, x_{0} \cdot x_{1} \cdots \cdots x_{p-1} \in\left(C\left\langle x_{p}\right\rangle, D\right)$. Thus $x_{0} \cdot x_{1} \cdots \cdots x_{p} \in\left(C\left\langle x_{p}\right\rangle, D\right)$. Therefore

$$
\left(C\left\langle x_{0} \cdot x_{1} \ldots \ldots x_{p}\right\rangle, D\right) \subset\left(C\left\langle x_{p}\right\rangle, D\right) \subset\left(C\left(x_{0}, x_{1}, \ldots, x_{p}\right), D\right) .
$$

7. Imbedding of $\left(C\left(x_{0}, x_{1}, \ldots, x_{p}\right), D\right)$ in a graduated logarithmic field $\left({ }^{7}\right)$. Let $K, C, D$ and the logarithmic sequence $x_{0}, x_{1}, \ldots$ be as in $\$ 2$. Recall that $K$ is a differential field with derivative $D, C$ is the subfield of constants: $C=\{c: c \in K$ such that $D c=0\}$, and $x_{0}, x_{1}, \ldots$ is a logarithmic sequence in $K$. Suppose further that $K$ contains a multiplicative subgroup $U^{\prime}$ such that for every $f \in U^{\prime}$ and every integer $r \geqq 1$ there is a unique $g \in U^{\prime}$ such that $g^{r}=f$; we will denote $g$ by $f^{1 / r}$. Furthermore, suppose $U^{\prime}$ contains the set $\left\{x_{0}, x_{1}, \ldots\right\}$. Let $U_{p}$ be the subgroup of $U^{\prime}$ generated by the elements of the form $x_{i}^{m} \in U^{\prime}$, where $m$ is rational and $0 \leqq i \leqq p$. Let $G_{p}$ be the differential subfield of $K$ generated by $U_{p}$ over $C$. We observe that $\left(C\left(x_{0}, x_{1}, \ldots, x_{p}\right), D\right)$ is a differential subfield of $\left(G_{p}, D\right)$. Moreover, $\left(G_{p}, D\right)$ is an algebraic extension of $\left(C\left(x_{0}, x_{1}, \ldots, x_{p}\right), D\right)$. Since the transcendence degree of $\left(C\left(x_{0}, x_{1}, \ldots, x_{p}\right), D\right)$ over $C$ is $p+1$ by Lemma 2.2 , the transcendence degree of $\left(G_{p}, D\right)$ is $p+1$ over $C$. Let $(G, U, D)=\lim _{p \rightarrow \infty}\left(G_{p}, U_{p}, D\right)$. It is clear that $(G, D)$ is a differential field.

We will introduce for the differential field $\left(G_{p}, D\right)$ a partial order $<$, whose restriction to the differential subfield $\left(C\left(x_{0}, x_{1}, \ldots, x_{p}\right), D\right)$ coincides with the partial order < defined on $\left(C\left(x_{0}, x_{1}, \ldots, x_{p}\right), D\right)$ in $\S 3$, and which is such that the quadruple $\left(G_{p},<, U_{p}, C\right)$ is a graduated field as defined in [7]. Define the order relation $<$ in $U_{p}$ as follows. Let $\{M, N\} \subset U_{p}$, then $M=x_{0}^{m_{0}} \cdot x_{1}^{m_{1}} \cdots \cdots x_{p}^{m_{p}}$ and $N=x_{0}^{n} 0 \cdot x_{1}^{n_{1}} \cdots \cdots x_{p^{p}}^{n}$ where the exponents are rational numbers. Set $M<N$ if $m_{0}<n_{0}$, or if for some natural number $q, 0<q \leqq p, m_{k}=n_{k}$ for $k=0,1, \ldots, q-1$ and $m_{q}<n_{q}$. Let $\mathscr{M}=C^{*} \cdot U_{p}$ where $C^{*}=C-\{0\}$. Define the order relation in $\mathscr{M}$ as follows. If $\left\{g^{*}, h^{*}\right\} \subset \mathscr{M}$ then $g^{*}=c M$ and $h^{*}=d N$ for some $M$ and $N$ in $U_{p}$ such that $c, d \in C^{*}$. Set $g^{*}<h^{*}$ if $M<N$ and $g^{*} \approx h^{*}$ if $M=N$. It is clear that the order

( 7 ) The graduated differential field $(X, D)$ (see Lemma 7.3) is a graduated logarithmic field if $U$ contains a logarithmic sequence (see also [8, p. 14]). 
relation $<$ in $\mathscr{M}$ is compatible with the order relation $<$ in $U_{p}$. We now define an order relation $<$ in $G_{p}-\{0\}$. Suppose first that $f \in G_{p}-\{0\}$ and $f=\sum_{i=1}^{n} c_{i} N_{i}$ with $c_{i} \in C^{*}$ and $N_{i} \in U_{p}$ (with $i \neq j \Rightarrow N_{i} \neq N_{j}$ ), then for some $r, c_{i} N_{i} \lesssim c_{r} N_{r}$ for $i=1$, $2, \ldots, n$, with strict inequality if $i \neq r$. The representation $f=\sum_{i=1}^{n} c_{i} N_{i}$ is unique. In fact for an arbitrary nonzero $p+1$-tuple $\left(r_{0}, r_{1}, \ldots, r_{p}\right)$ of rational numbers, $x_{0}^{r_{0}}, x_{1}^{r_{1}}, \ldots, x_{p}^{r_{p}}$ are algebraically independent over $C$. In this case we write $f \sim c_{r} N_{r}$, and we say $c_{r} N_{r}$ is the dominating monomial of $f$. If now $f$ is any element of $G_{p}-\{0\}$, then $f=\sum c_{i} M_{i} / \sum d_{j} N_{j}$. Let $g^{*}$ and $h^{*}$ be the dominating monomials of the numerator and the denominator respectively. We write $g^{*} \cdot h^{*-1} \sim f$, and call $g^{*} \cdot h^{*-1}$ the dominating monomial of $f$. Let $(c, u)$ be the unique element of $C \times U_{p}$ such that $g^{*} \cdot h^{*-1}=c u$. Then $u$ is called the gauge of $f$ and is denoted by $] f\left[\left({ }^{8}\right)\right.$. If $f_{1}$ and $f_{2}$ belong to $G_{p}-\{0\}$, we say $f_{1}<f_{2}$ if and only if $] f_{1}[<] f_{2}\left[\right.$. If $f_{1}^{*}$ and $f_{2}^{*}$ are the dominating monomials of $f_{1}$ and $f_{2}$ respectively and $f_{1}^{*}=f_{2}^{*}$, then we write $f_{1} \sim f_{2}$. It is clear that this order relation in $G_{p}-\{0\}$ is compatible with the order relation in $\mathscr{M}$. We extend this definition of order by setting $0<f$ for every $f \in G_{p}-\{0\}$, and $] 0\left[=0\right.$. Thus $f_{1} \sim f_{2}$ if and only if $f_{1}^{*}=f_{2}^{*}$, and hence if and only if $f_{1}-f_{2}<f_{2}$. It is now clear that the partial order < defined here restricted to the subfield $C\left(x_{0}, x_{1}, \ldots, x_{p}\right)$ of $G_{p}$ coincides with the partial order < defined in $\S 3$. We observe that this partial order can be extended to the differential field $(G, D)$.

LEMMA 7.1. If $M \in U_{p}-\{1\}$ and $e \in U_{p}$, such that $e<1$, then $M D e<D M$.

Proof. If $M=x_{n}^{m} \cdot x_{n+1}^{m_{1}} \cdots \cdots x_{p^{p-n}}^{m_{1}}$ with $m \neq 0$, then by routine calculations $m\left(x_{0} \cdot x_{1} \cdots \cdots x_{n}\right)^{-1} \sim M^{-1} D M$. On the other hand $e=x_{m}^{b} \cdot x_{m+1}^{b_{1}} \cdots \cdots x_{p}^{b_{p}-m}$ with $b<0$. Then $D e \sim b\left(x_{0} \cdot x_{1} \cdots \cdots x_{m}\right)^{-1} \cdot e<M^{-1} D M$ by lexicographic comparison of exponents. Thus $M D e<D M$.

LEMMA 7.2. If $\{M, N\} \subset U_{p}, N \neq 1$, such that $M<N$, then $D M<D N$.

Proof. $M<N$ implies $M=N e$ for some $e \in U_{p}$ with $e<1$. Hence $D M=e D N$ $+N D e$. Now $e D N<D N$ since $e<1$ and $N D e<D N$ by Lemma 7.1. Thus $D M<D N$.

LEMMA 7.3. Let $G_{p}, G, D, C, U_{p}, U$, < and the logarithmic sequence $\left\{x_{0}, x_{1}, \ldots\right\}$ be as defined above, then

(i) the ordered pairs $\left(G_{p},<\right),(G,<)$ are fields with asymptotic order (for the definition of asymptotic order (see [7, p. 231])).

(ii) the ordered quadruples $X_{p}=\left(G_{p},<, U_{p}, C\right)$ and $X=(G,<, U, C)$ are graduated fields (see [7, p. 231]).

(iii) $\left(X_{p}, D\right)$ is a graduated differential field. This means $D C=\{0\}$ and $D$ is stable at $U_{p}-\{1\}\left(^{9}\right)$. (See also [8, Definition 20].)

(iv) $(X, D)$ is a graduated logarithmic field.

(8) See Definition 17 of [7].

( ${ }^{9} D$ is stable at $U_{p}-\{1\}$ if $M<N$ implies $D M<D N$ whenever $M \in G_{p}$ and $N \in U_{p}-\{1\}$ (see also [8, Definition 18]). 
Proof. (i) and (ii) follow from the order relation defined in $G_{p}$. To show (iii) it is enough to show $D$ is stable on $U_{p}-\{1\}\left(^{9}\right)$. This follows from Lemma 7.2. (iv) is obvious.

LEMMA 7.4. Let $y \in\left(C\left(x_{0}, x_{1}, \ldots, x_{p}\right), D\right)$. Let $m$ be a nonzero rational number. Then $(C\langle y\rangle, D)$ and $\left(C\left\langle y^{m}\right\rangle, D\right)$ have the same transcendence degree over $C$.

Proof. Suppose $m=a / b$ with $a$ and $b$ integers, $b>0$. Then $(C\langle y\rangle, D)$ is an algebraic extension of $\left(C\left\langle y^{a}\right\rangle, D\right)$. Hence the transcendence degree of $\left.\left(C<y^{a}\right\rangle, D\right)$ is same as the transcendence degree of $(C\langle y\rangle, D)$. Similarly $\left(C\left\langle y^{a / b}\right\rangle, D\right)$ is an algebraic extension of $\left(C\left\langle y^{a}\right\rangle, D\right)$. Thus $\left(C\left\langle y^{m}\right\rangle, D\right),\left(C\left\langle y^{a}\right\rangle, D\right)$ and $\left.(C<y\rangle, D\right)$ have the same transcendence degree over $C$.

THEOREM 7.1. Let $m$ be a nonzero rational number, then

(i) the transcendence degree of $\left(C\left\langle\left(x_{0} \cdot x_{1} \cdots \cdots x_{p}\right)^{m}\right\rangle, D\right)$ is $p+1$ over $C$.

(ii) the transcendence degree of $\left(C\left\langle x_{p}^{m}\right\rangle, D\right)$ is $p+1$ over $C$.

(iii) let $V_{p}=\left(x_{0} \cdot x_{1} \cdots \cdots x_{p}\right)^{m}$, then $V_{p}, D V_{p}, \ldots, D^{p} V_{p}$ are algebraically independent over $C$.

(iv) $x_{p}^{m}, D x_{p}^{m}, \ldots, D^{p} x_{p}^{m}$ are algebraically independent over $C$.

(v) $V_{p}$ and $x_{p}^{m}$ satisfy no algebraic differential equation of order less than $p+1$.

Proof. (i) and (ii) follow from Theorems 6.1 and 6.2 and Lemma 7.4 above. (iii) and (iv) follow from (i) and (ii). (v) follows from (iii) and (iv).

\section{Applications.}

Definition $8.1\left({ }^{10}\right)$. Let $P$ be the algebraic differential operator defined by

$$
P(y)=\sum a_{i} y^{i_{0}}(D y)^{i_{1}} \ldots \ldots\left(D^{p} y\right)^{i_{p}}, \quad a_{i} \in C .
$$

We say $P$ is homogeneous of degree $d$ if $i_{0}+i_{1}+\cdots+i_{p}=d$, and is isobaric of weight $W$ if $i_{1}+2 i_{2}+\cdots+p i_{p}=W$ for every monomial effectively present in the right side of formula (I).

LEMMA 8.1. Let $P$ be the algebraic differential operator given by formula (I) above. Let $d$ and $W$ be positive integers. Let $P$ be homogeneous of degree $d$ and isobaric of weight $W$. Let $m$ be a rational number and $q=d m-W$. Then under the substitution $y=x_{0}^{m} \cdot z$, the expression $x_{0}^{-a} \cdot P(y)$ is transformed into

$$
Q(z)=\sum b_{j} z^{j_{0}}\left(x_{0} D z\right)^{j_{1}} \ldots \cdots\left(\left(x_{0} D\right)^{p} z\right)^{j_{p}}
$$

where $b_{j} \in C$.

Proof. By formula 66(c) of [8], or induction on $k$

$$
D^{k}\left[x_{0}^{m} z\right]=x_{0}^{m-k} \cdot \sum_{i=0}^{k} S_{k, i}(m)\left(x_{0} D\right)^{k-i} \cdot z
$$

$\left({ }^{10}\right)$ See $\$ \$ 2-4$ of [8]. 
for any positive integer $k$. Substitution of $y=x_{0}^{m} z$ in $P(y)$ with the aid of formula (II) establishes the Lemma.

DEFINITION 8.2. The operator $Q$ of Lemma 8.1 will be called the m-image of the operator $P$ given by the formula (I).

THEOREM 8.1 (STRODT). Under the hypotheses of Lemma 8.1, if $m$ is nonzero (rational), then $Q$ effectively involves terms of weight less than $W$.

Note. This theorem was proven by W. Strodt in the general context of a graduated logarithmic field (cf. [8, \$69]). The proof in [8] depends upon the analytic proof of a special case of this theorem $[6, \S 61]$. We eliminate here this dependence upon the analytic proof.

Proof of Theorem 8.1. $P(Y)=\sum a_{i} Y^{i_{0}}(D Y)^{i_{1}} \ldots \ldots\left(D^{p} Y\right)^{i_{p}}$ and

$$
\begin{aligned}
Q(Y) & =\sum a_{i} Y^{i_{0}} \cdot\left(x_{0} D Y+S_{11}(m) Y\right)^{i_{1}} \ldots \ldots\left(\left[x_{0} D\right]^{p} Y+\sum_{i=1}^{p} s_{p, i}(m)\left[x_{0} D\right]^{p-i} Y\right)^{i_{p}} \\
& =P\left(Y, x_{0} D Y, \ldots,\left[x_{0} D\right]^{p} Y\right)+H\left(Y, x_{0} D Y, \ldots,\left[x_{0} D\right]^{P} Y\right)
\end{aligned}
$$

where

$$
P\left(Y, x_{0} D Y, \ldots,\left[x_{0} D\right]^{p} Y\right)=\sum a_{i} Y^{i_{0}}\left(x_{0} D Y\right)^{i_{1}} \ldots \ldots\left(\left[x_{0} D\right]^{p} Y\right)^{i_{p}} .
$$

It is clear that all the terms of $H\left(Y, x_{0} D Y, \ldots,\left[x_{0} D\right]^{p} Y\right)$ have coefficients in $C$ and are of weight less than $W$. Suppose $H\left(Y, x_{0} D Y, \ldots,\left[x_{0} D\right]^{p} Y\right) \equiv 0$, then

$$
Q(Y) \equiv P\left(Y, x_{0} D Y, \ldots,\left[x_{0} D\right]^{p} Y\right) .
$$

If $y \in C\left(x_{0}, x_{1}, \ldots, x_{k}\right), k<p$, then

$$
\begin{aligned}
P\left(E y, x_{0} D E y, \ldots,\left[x_{0} D\right]^{p} E y\right) & =P\left(E y, E D y, \ldots, E D^{p} y\right) \\
& =E P(y) \text { by Lemma 5.1, }
\end{aligned}
$$

where $E y\left(x_{0}, x_{1}, \ldots, x_{k}\right)=y\left(x_{1}, x_{2}, \ldots, x_{k+1}\right), \quad($ Definition 3.1). Since $W>0$, $P(1)=0$ hence

$$
\begin{aligned}
Q(1)=x_{0}^{-d m+w} P\left(x_{0}^{m}\right) & =P\left(1, x_{0} D 1, \ldots,\left[x_{0} D\right]^{p} 1\right) \text { by (III) } \\
& =0 .
\end{aligned}
$$

Thus $P\left(x_{0}^{m}\right)=0$. Now suppose that for $k>0, P\left(\left(x_{0} \cdot x_{1} \cdots x_{k-1}\right)^{m}\right)=0$. Then

$$
\begin{aligned}
x_{0}^{-d m+w} P\left(\left(x_{0} \cdot x_{1} \cdots \cdots x_{k}\right)^{m}\right) & =Q\left(\left(x_{1} \cdot x_{2} \cdots \cdots x_{k}\right)^{m}\right) \\
& =P\left(\left(x_{1} \cdot x_{2} \cdots \cdots x_{k}\right)^{m}, x_{0} D\left(x_{1} \cdot x_{2} \cdots \cdots x_{k}\right)^{m}, \ldots,\right. \\
& \left.\quad\left[x_{0} D^{p}\right]\left(x_{1} \cdot x_{2} \cdots \cdots x_{k}\right)^{m}\right) \text { by (III) } \\
& =E P\left(\left(x_{0} \cdot x_{1} \cdots \cdots x_{k-1}\right)^{m}\right) \quad \text { by (IV) } \\
& =0 .
\end{aligned}
$$

It thus follows by induction that $P\left(\left(x_{0} \cdot x_{1} \cdots \cdots x_{p}\right)^{m}\right)=0$. This contradicts Theorem 7.1(v) and completes the proof. 
THEOREM 8.2 (S. BANK). Under the hypothesis of Lemma 8.1 if $m=0$ and $P(Y) \neq c Y^{d-w} \cdot(D Y)^{w}, c \in C-\{0\}$, then $Q$ effectively involves terms of weight less than $W$.

Note. In the case where $C$ is the field of complex numbers and $x_{0}=x, x_{1}$ $=\log x, \ldots, x_{p}=\log x_{p-1}$, this theorem has been proven by S. Bank [1, Lemma 13].

Proof of Theorem 8.2. As in the proof of the previous theorem

$$
Q(Y)=P\left(Y, x_{0} D Y, \ldots,\left[x_{0} D\right]^{p} Y\right)+H\left(Y, x_{0} D Y, \ldots,\left[x_{0} D\right]^{p} Y\right) .
$$

If we suppose $P(Y)=c Y^{d-w} \cdot(D Y)^{w}+T(Y)$ where $T(Y) \not \equiv 0$ and has its coefficients in $C$ and is homogeneous of degree $d$, isobaric of weight $W$ and is of order $\geqq 2$, then $Q(Y)=c Y^{d-w} \cdot\left(x_{0} D Y\right)^{w}+R\left(Y, x_{0} D Y, \ldots,\left[x_{0} D\right]^{p} Y\right)$ where $R$ is the 0 -image of $T(Y)$ (see Definition 8.2). If the conclusion of the theorem is assumed false, then $H\left(Y, x_{0} D Y, \ldots,\left[x_{0} D\right]^{p} Y\right) \equiv 0$. Thus

$$
T\left(Y, x_{0} D Y, \ldots,\left[x_{0} D\right]^{p} Y\right) \equiv R\left(Y, x_{0} D Y, \ldots,\left[x_{0} D\right]^{p} Y\right) .
$$

Since every term of $T(Y)$ is of order $\geqq 2, T\left(x_{0}\right)=0$. Now suppose that for $k>0$, $T\left(x_{k-1}\right)=0$. Then

$$
\begin{aligned}
x_{0}^{W} T\left(x_{k}\right) & =R\left(x_{k}, x_{0} D x_{k}, \ldots,\left[x_{0} D\right]^{p} x_{k}\right) \\
& =T\left(x_{k}, x_{0} D x_{k}, \ldots,\left[x_{0} D\right]^{p} x_{k}\right) \quad \text { by }(\mathrm{V}) \\
& =E T\left(x_{k-1}\right) \text { by (IV) } \\
& =0 .
\end{aligned}
$$

It follows by induction that $T\left(x_{p}\right)=0$. This contradicts Theorem 7.1(v) and establishes the theorem.

\section{REFERENCES}

1. S. Bank, On the instability theory of differential polynomials, Ann. Mat. Pura Appl. (4) 74 (1966), 83-112.

2. E. R. Kolchin, Extensions of differential fields. I, Ann. of Math. (2) 43 (1942), 724-729.

3. - Differential algebra (to appear).

4. I. Kaplansky, An introduction to differential algebra, Hermann, Paris, 1957.

5. J. F. Ritt, Differential algebra, Amer. Math. Soc. Colloq. Publ., Vol. 33, Amer. Math. Soc., Providence, R. I., 1950; reprint, Dover, New York, 1966.

6. W. Strodt, Contributions to the asymptotic theory of ordinary differential equations in the complex domain, Mem. Amer. Math. Soc. No. 13 (1954).

7. - On the algebraic closure of certain partially ordered fields, Trans. Amer. Math. Soc. 105 (1962), 229-250.

8. —_ Graduated logarithmic fields and stability, Univ. Wisconsin MRC Tech. Summary Rep. No. 489, 1964.

9. O. Zariski and P. Samuel, Commutative algebra, Vol. 1, Van Nostrand, Princeton, N. J., 1958.

UNIVERSITY OF ILLINOIS, URBANA, ILLINOIS 